\title{
Selection Biases in Likelihood Arguments
}

\author{
Matthew Kotzen \\ University of North Carolina at Chapel Hill \\ Department of Philosophy
}

Draft of May 17, 2009

\section{Introduction}

It has impressed many physicists and philosophers that various features of our universe appear to be "fine-tuned" for life. Take, for example, the value of the gravitational constant of the universe, G. It is claimed that if $\mathrm{G}$ had taken a value that was more than a little bit higher than its actual value, the universe would have collapsed back in on itself nanoseconds after the Big Bang, and the universe would have been too hot and dense for life to ever evolve. Similarly, if $\mathrm{G}$ had taken a value that was more than a little bit lower than its actual value, the universe would have spread out so quickly that the heavy elements would have never been able to form, and the universe would have consisted entirely of hydrogen and helium, which again would not have allowed for life to evolve.

Of course $\mathrm{G}$ could have taken a value that was a little bit different from its actual value and life would still have been possible. But many people have been struck by the relative narrowness of the range of "life-sustaining" values that G could have taken, at least compared with all the metaphysically possible values that $\mathrm{G}$ could have taken.

In this paper, I will rehearse in Section 2 a version of the "Fine-Tuning Argument" (FTA) that appeals to the considerations above in order to argue for the existence of a Designer who is interested in seeing to it that life is possible. In Section 3, I will present the Objection from Anthropic Bias to the FTA. In Section 4, I will raise some problems for the Objection from Anthropic Bias, and in Section 5, I will present and defend my own analysis of the FTA. My

conclusion will be that the Objection from Anthropic Bias is not a successful response to the FTA.

\section{The Fine-Tuning Argument}

As it is usually presented, the FTA relies on the

Likelihood Principle (LP): $E$ is evidence for $H 1$ over $H 2$ iff $p(E \mid H 1)>$ $p(E \mid H 2)$ 
In the FTA, we use the following abbreviations:

$E=$ "The constants of the universe have life-sustaining values."

$D=$ "An intelligent designer set the values of the constants."

$C=$ "The values of the constants were set by chance."

According to the FTA, $p(E \mid D)>p(E \mid C)$, so $E$ is evidence for $D$ over $C$ by LP.

Why, according to the FTA, is $p(E \mid D)>p(E \mid C)$ ? First, it seems that $p(E \mid C)$ has to be very very low, since it seems to be extraordinarily unlikely that the values of the constants would just happen to fall in the narrow life-sustaining range if those constants were set by chance; after all, the vast "majority" 11 of the metaphysically possible values that the constants could take are not lifesustaining. By contrast, if there is a Designer who set the values of the constants, it seems to be rather more probable that that Designer would intentionally see to it that the constants were life-sustaining, since universes containing observers seem to be the sort of thing that Designers would (or, at least, plausibly might) intentionally aim at.

In order for the FTA to go through, it's not required that $p(E \mid D)$ be high-in other words, it's not required that we think that an intelligent designer would be especially likely to set the constants to life-sustaining values. All that is required for the FTA to go through is that the constants are more likely to take on lifesustaining values if $D$ is true than if $C$ is true - in other words, that a Designer is more likely to set the constants to life-sustaining values than some undirected chance process is to result in constants with life-sustaining values. Since this has seemed very plausible, it has seemed plausible that $p(E \mid D)>p(E \mid C)$, and so, by LP, that $E$ is evidence for $D$ over $C$.

There are a number of reactions to the FTA that have been defended, but I will focus on only one - the "Objection from Anthropic Bias"-in this paper. Let me mention a few of the other reactions just to set them aside.

Some philosophers have reacted to the FTA by pointing out that we have only a rough idea of how "robust" life and the evolutionary process that produced life really are. We have a pretty good idea of what is required for lifeforms sufficiently like us to evolve. But we have only the faintest idea of what other sorts of observation-capable lifeforms might be able to exist in very different universes. Perhaps, for example, observation-capable life could exist in universes consisting only of hydrogen and helium, so that the range of life-sustaining constants isn't nearly as narrow as we thought it was. I myself am doubtful of this reaction, but I agree that if we acquired good reason to think that observationcapable life could exist in universes with very different physical constants, then

\footnotetext{
${ }^{1}$ Of course I don't really mean "majority," since there are an infinite number of lifesustaining values of the constants and an infinite number of non-life-sustaining values of the constants. What I do mean is that there seems to some relevant measure according to which the measure of the life-sustaining values of the constants is dramatically lower than the measure of the non-life-sustaining constants.
} 
Section 3. The Objection from Anthropic Bias

that would be an effective objection to the FTA. I just don't think that we yet have any such good reason.

Other philosopher $2^{2}$ have suggested that there's a technical problem with assigning probabilities to outcomes in the space of all metaphysically possible values for the constants. They point out that probability distributions only make sense when the relevant space is "normalizable"-i.e., where there's a way to assign probabilities to mutually exclusive outcomes such that the sum of those probabilities is 1 . I think that too much is being made out of a technical point here; I think that a booming voice from the clouds which made lots of accurate predictions about the future would be some evidence for $D$, even though I have no idea how to best normalize the space of possible things that a Designer might do.

Third, some philosophers ${ }^{3}$ have suggested that we'd need to have insight into the Designer's psychology in order to rationally believe that $p(E \mid D)>p(E \mid C)$ in other words, that we'd have to have reason (which we don't in fact have) to believe that a Designer is more likely to be the sort of being who is interested in bringing about a universe with observers than one who is interested in bringing about a universe full of hydrogen and helium, say. But since none of us is in a position to do "divine psychology," these philosophers continue, the FTA can't go through. Again, I think it's clear that the "booming voice from the clouds" would be evidence for $D$ over $\neg D$, and since LP is a biconditional, that entails that the booming voice from the clouds is likelier on the supposition of $D$ than on the supposition of $\neg D$-in other words, that a Designer is more likely to produce a booming voice from the clouds than a Designer-less world is. In some sense, this does commit me to thinking that we can have some insight into divine psychology, but I don't find this consequence particularly troubling.

Fourth, a number of philosophers 4 have been interested in the question of whether $E$ provides evidence for the hypothesis $M$ that there are lots of other universes besides this one, each with their own sets of constants. The idea here is that, while it would be very surprising for the only universe to just happen to have life-sustaining constants, it would be rather less surprising for one among many universes to have life-sustaining constants. Though there may be some interesting connections between the question of whether $E$ is evidence for $D$ and whether $E$ is evidence for $M, \mathrm{I}$ am going to ignore those questions in what follows. My interest in this paper is only in whether one particular response to the FTA from $E$ to $D$ is successful.

\section{The Objection from Anthropic Bias}

The objection to the FTA that I will be discussing is the charge that the FTA ignores a certain type of observation bias - namely, the bias that is constituted by the fact that intelligent observers like us can exist only in life-sustaining

\footnotetext{
${ }^{2}$ See McGrew et al. 2001 and Garfield et al. 2005.

${ }^{3}$ See Sober 2002 and Sober 2004.

${ }^{4}$ See, e.g., Manson and Thrush 2003, Juhl 2005, and White 2000.
} 
universes. As a result of this bias, the objection continues, the fact that we observe a life-sustaining universe is no evidence at all for $D$, since (given that we exist) we were guaranteed to observe a life-sustaining universe.

Let's try to make this objection more precise. The thought behind the objection is that observers like us can observe only life-sustaining universes, since the universe's being life-sustaining is a pre-condition for the existence of observers like us. And it's no evidence for anything (and a fortiori no evidence for $D$ over $C$ ) when we observe a pre-condition for our own existence.

So, the Objection from Anthropic Bias is claiming that we're failing to take account of all of the relevant information when we judge that $p(E \mid D)>p(E \mid C)$. But according to the Requirement of Total Evidence (RTE), we should always take account of the strongest statement of our evidence that we have available to us.

For example, suppose that I know that a friendly bear is running toward me. That is no evidence that I'm about to die, since I know that friendly bears don't ever hurt people. But if instead of considering the fact that there's a friendly bear running toward, I had instead considered only the (true) fact that there's a bear running toward me, that would be evidence that I'm about to die, since bears harm people all the time.

What information am I failing to take account of, according to the Objection from Anthropic Bias, when I judge that $p(E \mid D)>p(E \mid C)$ ? The answer is the information that I am alive at $t$ (some time after my birth), observing whether the constants are life-sustaining. And, of course, if I'm around at $t$ observing whether the constants are life-sustaing, then I'm certain to observe that they are indeed life-sustaining, since I couldn't exist in a universe without life-sustaining constants.

An analogy that gets invoked frequently here in the fine-tuning literature is one from Eddington ${ }^{5}$ Suppose that there are a bunch of fish in a pond, and I'm wondering whether the pond consists of mostly large fish $(L)$ or mostly small fish $(S)$. Suppose that I have a net that can catch only one fish at a time, and suppose that there are enough fish in the pond so that I am certain to catch one fish each time I throw my net into the pond.

Now, I throw my net into the pond at $t$ and catch a large fish $(E)$. According to LP, this is some evidence for $L$, since $p(E \mid L)>p(E \mid S)$, and that seems like the right result. But then suppose that I look down at my net and see that the holes in my net are too large to catch small fish $(I)$. Once I notice this, it would be epistemically irresponsible for me to ignore it and take myself to still have evidence for $L$ since $p(E \mid L)>p(E \mid S)$. Rather, the relevant question seems to be whether $p(E \mid L \wedge I)>p(E \mid S \wedge I)$-in other words, whether I was likelier to catch a large fish if the pond consisted of mostly large fish and the holes in my net are too large to catch small fish or if the pond consisted of mostly small fish and the holes in my net are too large to catch small fish. And here, it's clear that $p(E \mid L \wedge I)=p(E \mid S \wedge I)$, which gives us the correct result that $E$ is no evidence for $L$ over $S$ once we know $I$.

\footnotetext{
${ }^{5}$ See Eddington 1939.
} 
Section 4. Problems with the Objection from Anthropic Bias

The lesson of Eddington's case is often implicitly taken to be that LP needs to be amended (or perhaps just precisified) to something like:

Likelihood Principle* (LP*): Where $I$ is our information about the process of observation, $E$ is evidence for $H 1$ over $H 2$ iff $p(E \mid H 1 \wedge I)>p(E \mid H 2 \wedge I)$

In Eddington's case, given that I'm using a net with holes too large to catch a small fish, I'm just as likely to catch a large fish in my net regardless of whether the $L$ or $S$ is true, so $p(E \mid L \wedge I)=p(E \mid S \wedge I)$. So, by applying LP* in Eddington's case, we get the correct result that $E$ is no evidence for $L$ over $S$.

The application of $\mathrm{LP}^{*}$ to the FTA is as follows. Just as $I$ included the fact that the holes in my net are too large to catch small fish in Eddington's case, it seems that in the FTA, $I$ should include the fact that I'm around at $t$ to make observations, since that is part of the information that we have about the relevant process of observation. So the relevant question to ask in the FTA isn't whether $p(E \mid D)>p(E \mid C)$ (as LP recommended), but rather whether $p(E \mid D \wedge I)>p(E \mid C \wedge I)$ (as LP* recommends). But it's fairly obvious that if $I$ includes the information that I'm around at $t$ to make observations, then $p(E \mid D \wedge I)=p(E \mid C \wedge I)=1$, since if I'm around at $t$ to make observations, I'm certain to observe life-sustaining constants regardless of whether $D$ or $C$ is true. Thus, according to the Objection from Anthropic Bias, the FTA can be seen to be bad once we see that we need to respect the RTE and take into consideration our full information about the relevant process of observation, and once we see that we need to move from LP to LP* in order to take appropriate account of that information.

\section{Problems with the Objection from Anthropic Bias}

\subsection{The Line-Drawing Problem}

According to LP*, when calculating likelihoods, we have to assume information $I$ about the relevant process of observation "on the right"-i.e., by conjoining $I$ to the expression on the right-hand side of the conditionalization bar. Moreover, in order to respect the RTE, I has to be our total evidence about the relevant process of observation; if we leave any information about the process of observation out, we will make the same mistake as if I took a friendly bear running toward me to be evidence that I am about to die.

But this naturally leads us to wonder: how total should our "total evidence" about the process of observation be? We learned from Eddington's fish case that $I$ needs to include facts about the size of the holes in the net that I use. But another fact about the process of observation in Eddington's fish case is that the process of observation results in my catching a large fish. That's a fact about the process of observation too, so if the information that the net has large holes is included in $I$, it's not at all obvious why the information that the 
process of observation resulted in my catching a large fish shouldn't be included as well.

But this would be disastrous, and would completely trivialize LP*. For consider a variant of the Eddington fish case where the net that I use has holes small enough to catch small fish, so that $E$ really is evidence for $L$ over $S$. In this case, if we include only the information that the net has small holes in $I$, then LP* gives us the right result that since $p(E \mid L \wedge I)>p(E \mid S \wedge I), E$ is evidence for $L$ over $S$. But if we also include in $I$ the information that the process of observation resulted in my catching a large fish, then LP* gives us the wrong result that $E$ is no evidence for $L$ over $S$, since then $p(E \mid L \wedge I)=$ $p(E \mid S \wedge I)=1$; after all, given that my net has small holes and that I catch a large fish, I'm certain to catch a large fish regardless of whether $L$ or $S$ is true. More generally, whenever we include information in $I$ which entails $E$, it will follow that $p(E \mid H 1 \wedge I)=p(E \mid H 2 \wedge I)$, so that $E$ will never be any evidence for any hypothesis over any other hypothesis, which is absurd.

So, LP* leads us into a puzzle: How much information about the process of observation ought to be included in $I$ ? We have to include in $I$ the information that the net has large holes, but we can't include in $I$ the information that the process of observation results in the collection of $E$. It's somewhat hard to see how to draw this line between the information about the process of observation that should be included in $I$ and the information that shouldn't be included in $I$ in a principled way, and the burden seems to be on the defender of LP* to do this. Call this the Line-Drawing Problem.

There have been attempts to solve the Line-Drawing Problem (though not presented in these terms) in the literature on fine-tuning. In particular, Sober ${ }^{6}$ and Weisberg7 have defended views about how much information ought to be included in $I$; Sober thinks that all of the information about the process of observation should be included ${ }^{8}$ whereas Weisberg thinks that only conditionals about what you could not have observed should be included in $I$. I don't intend to enter into this debate here, as I think the debate is misguided. But the Line-Drawing Problem is not a mere hypothetical one; it is a problem that philosophers who assume LP* have already spent time trying to solve.

\subsection{Firing Squad Cases}

A second problem with LP* is that there are cases which seem to be relevantly like the FTA, but where including information analogous to "I'm around at $t$ making observations" in $I$ gets us the wrong results. This puts pressure on the defender of the Objection from Anthropic Bias to explain why "I'm around at $t$ making observations" should be included in $I$ in the FTA, whereas the analogous information shouldn't be included in $I$ in these cases.

\footnotetext{
${ }^{6}$ Sober 2009.

${ }^{7}$ Weisberg 2005.

${ }^{8}$ Sober doesn't specifically address the issue of whether the information that the process of observation resulted in the collection of $E$ should be included in $I$; I assume that he doesn't think that it should, but I don't know how he solves the Line-Drawing Problem.
} 
Section 4. 4.3 Probabilistic Dependence Between $I$ and the Hypotheses

Here's one such case $9^{9}$ Suppose that I'm in front of a firing squad, and I know that the shooters are either going to intentionally miss me $(D)$ or that they are are going to fire at random in my general direction $(C)$. Intuitively, when I observe at $t$ (a moment after the shooting) that I'm alive, that is some evidence for $D$ over $C$, since I was much more likely to survive the firing squad on the assumption that the shooters intentionally missed me than I was on the assumption that the shooters shot at random (since it's fairly likely that at least one randomly shot bullet would fatally wound me). In other words, it's intuitive that my observation that I'm alive at $t(E)$ is evidence for $D$ over $C$ precisely because $p(E \mid D)>p(E \mid C)$. But if $\mathrm{LP}^{*}$ is true rather than LP, then that probabilistic inequality is irrelevant. Rather, what is relevant is whether $p(E \mid D \wedge I)>p(E \mid C \wedge I)$. And if, as the Objector from Anthropic Bias holds, "I'm around at $t$ to make observations" should be included in $I$ in the FTA, then it seems that that information should be included here too. But then $p(E \mid D \wedge I)=p(E \mid C \wedge I)=1$, since I'm certain to observe that I'm alive at $t$ given that I'm around at $t$ to make observations, regardless of whether $D$ or $C$ is true. Thus, LP* leads to the implausible result that my observing at $t$ that I'm alive is no evidence for $D$ over $C$.

Moreover, I don't think that this case essentially involves a situation where the relevant hypotheses affect my chances of surviving some ordeal, rather than my chances of coming to exist in the first place. Suppose that I know the following: just after the egg that was to become me was fertilized, doctors took that fertilized egg and placed it in a petri dish. It's not known what happened after that - either the doctors stored the fertilized egg carefully so as to make sure that it wouldn't be harmed $(D)$ or they threw it randomly around the lab in a way that made it very likely that the egg would be seriously damaged $(C)$. Again, it's quite intuitive that my existence at a time $t$ well after my birth $(E)$ is evidence for $D$ over $C$ because $p(E \mid D)>p(E \mid C)$. But again, if we accept LP* rather than LP, then that inequality is irrelevant; what is relevant is whether $p(E \mid D \wedge I)>p(E \mid C \wedge I)$, where $I$ presumably includes the information that I'm around at $t$ to make observations. But this inequality is obviously false; given that I'm around at $t$ to make observations, I'm certain to exist at $t$ regardless of whether $D$ or $C$ is true, so $p(E \mid D \wedge I)=p(E \mid C \wedge I)=1$. Again, LP* leads to the implausible result that $E$ is no evidence for $D$ over $C$.

\subsection{Probabilistic Dependence Between $I$ and the Hypothe- ses}

In the Firing Squad case from Section 4.2, the trouble with LP* seems to be that sometimes the information $I$ about the process of observation is itself evidence for one of the hypotheses under consideration, in virtue of itself being probabilistically non-independent of those hypotheses. In the Firing Squad case, the information $I$ that I'm around at $t$ to make observations is itself more likely on the supposition of $D$ than on the supposition of $C$, whereas that seemingly

\footnotetext{
${ }^{9}$ Weisberg 2005 makes use of this case.
} 
relevant fact gets ignored when we follow LP* and simply assume the truth of $I$ when evaluating the relevant likelihoods. Indeed, more generally, LP* entails the bizarre result that the contingent proposition $I$ can't ever be any evidence for any hypothesis, since $I$ is always being assumed by being conjoined to the expression on the right-hand side of the conditionalization bar.

In cases where $I$ is probabilistically independent of the relevant $H 1$ and $H 2$, as Eddington's fish case, LP* gives us the correct results. In Eddington's case, where $I=$ "I'm using a net with large holes," $I$ is probabilistically independent of $L$ and $S$; I'm no more or less likely to use a net with large holes on the supposition that $L$ is true than on the supposition that $S$ is true. By contrast, in the Firing Squad case, where $I$ is itself more likely on the supposition of $D$ than on the supposition of $C, \mathrm{LP}^{*}$ runs into trouble.

It's easy to change Eddington's fish case into one where $I$ fails to be probabilistically independent of $S$ and $L$. Imagine that the fish in the lake really want to get out of the lake for some reason, and imagine too that they have psychic powers which enable them to influence what sort of fish net I use (and imagine that I know all of this). If $L$ is true, the large fish in the lake will use their psychic powers to make it overwhelmingly likely that I'll use a net with large holes, and if $S$ is true, the small fish in the lake will use their psychic powers to make it overwhelmingly likely that I'll use a net with small holes. Now, suppose that I catch a large fish with a large-holed net. Here's a very bad argument that this provides me with no evidence for $L$ :

My process of observation included using a large-holed net $(I)$, so by LP* the relevant question is whether $p(E \mid L \wedge I)>p(E \mid S \wedge I)$. But here, $p(E \mid L \wedge I)=p(E \mid S \wedge I)$, since on the assumption that I'm using a large-holed net, I'm just as likely to catch a large fish regardless of whether $L$ or $S$ is true.

The problem with the argument above is that it ignores the probabilistic dependence between $I$ and $L$. Because of the background information that I have about the psychic powers of the fish, I know that the information $I$ that I used a large-holed net is itself evidence for $L$, even if that information also introduces a selection bias which affects how we should react to $E$.

Now, someone might well claim that I haven't identified a problem here with LP* itself, since LP* only characterizes the circumstances under which $E$ is evidence for $L$, and in the psychic fish case, it's $I$, not $E$, that is the real evidence for $L$. Now, I'm skeptical that we can make a clean distinction between whether $E$ or $I$ is "doing the real work" when the two are positively probabilistically correlated as they are in the psychic fish case, but my real response is that I'm happy to agree that once we know $I, E$ is no further evidence for $L$. But in the FTA, I don't really care whether it's $E$ or $I$ that constitutes evidence for $D$. In the FTA, I think that $E$ is evidence for $D$ before we take $I$ into consideration, and then once we do take $I$ into consideration, $I$ "blocks" the evidential effect of $E$, but only by a kind of "preemption" that occurs in virtue of $I$ itself being 
evidence for $D^{10}{ }^{11}$ Surely, the interesting and important question about the FTA is whether we should be more confident in $D$ after learning $E$ through a process of observation characterized by $I$, not whether it's $E$ or $I$ that deserves the epistemic "credit" for that increased confidence.

In the next section, I'll develop a proposal about how we should take account of information like $I$ that will avoid the problems that we encountered with LP*, and that will be explicitly designed to background this issue about whether $E$ or $I$ deserves the epistemic "credit" in any particular case.

\section{My Proposal}

Let's go back to the psychic fish case. If we're interested in the circumstances under which the collection of $E$ through a process characterized by $I$ is evidence for $L$, we can't use $p(E \mid L \wedge I)$ and $p(E \mid S \wedge I)$ as likelihoods, since those values will be equal, and will be insensitive to the probabilistic dependence between $L$ and $I$. Again, in the psychic fish case, it is relevant that $L$ makes $I$ very likely (and that $S$ makes $I$ very unlikely), so we might expect to somehow make use of a quantity like $p(I \mid L)$ and/or $p(I \mid S)$. Moreover, the fact that $L$ makes $I$ more probable than $S$ does increases the extent to which $L$ is confirmed over $S$, so we should expect the amount that $L$ is confirmed over $S$ to vary directly with $p(I \mid L)$ (or to vary inversely with $p(I \mid S)$ ).

My suggestion in the psychic fish case is that, instead of using $p(E \mid L \wedge I)$ and $p(E \mid S \wedge I)$ as likelihoods as LP* requires, we instead use $p(E \mid L \wedge I) \times$ $p(I \mid L)$ and $p(E \mid S \wedge I) \times p(I \mid S)$. These quantities clearly take account of the probabilistic dependence between $I, L$, and $S$ in the desired manner. And they clearly give us the correct result in the psychic fish case; as already discussed, $p(E \mid L \wedge I)=p(E \mid S \wedge I)$ in this case, so $p(E \mid L \wedge I) \times p(I \mid L)>p(E \mid S \wedge I) \times p(I \mid S)$ iff $p(I \mid L)>p(I \mid S)$, which is clearly true.

More generally, my proposal is:

Likelihood Principle** (LP**): The fact that $E$ was collected through a process of observation characterized by $I$ is evidence for $H 1$ over $H 2$ iff $p(E \mid H 1 \wedge I) \times p(I \mid H 1)>p(E \mid H 2 \wedge I) \times p(I \mid H 2)$

And it's easy to see that $p(E \mid H 1 \wedge I) \times p(I \mid H 1)=\frac{p(E \wedge H 1 \wedge I)}{p(H 1 \wedge I)} \times \frac{p(I \wedge H 1)}{p(H 1)}=$ $\frac{p(E \wedge H 1 \wedge I)}{p(H 1)}=p(E \wedge I \mid H 1)$ (and similarly that $p(E \mid H 2 \wedge I) \times p(I \mid H 2)=p(E \wedge$ $I \mid H 2))$. So, $\mathrm{LP}^{* *}$ can be equivalently stated as:

Likelihood Principle** (LP**): The fact that $E$ was collected through a process of observation characterized by $I$ is evidence for $H 1$ over $H 2$ iff $p(E \wedge I \mid H 1)>p(E \wedge I \mid H 2)$

In other words, LP** amounts to conjoining $I$ on the left of the conditionalization bar, rather than assuming $I$ on the right of the conditionalization bar as

\footnotetext{
${ }^{10}$ Which itself occurs because $I$ and $E$ are positively probabilistically correlated.

${ }^{11} \mathrm{I}$ also think the same thing is going on in the Firing Squad case.
} 
LP* requires. So LP** amounts to treating $I$ as "just another piece of evidence," to be treated just like $E$, and thus doesn't introduce a fundamental distinction between information that serves as evidence and information about the process of observation. And if $I$ is part of our evidence, as I think it is, then I have just as legitimate a claim to respecting the RTE by advocating LP** as the Objector from Anthropic Bias has to be respecting the RTE by advocating LP*.

In addition to the psychic fish case, $\mathrm{LP}^{* *}$ delivers the correct verdict in all of the uncontroversial cases that we've seen.

Consider Eddington's original fish case, without the psychic twist that I added. According to LP**, we should compare $p(E \mid L \wedge I) \times p(I \mid L)$ and $p(E \mid S \wedge$ $I) \times p(I \mid S)$. And since $I$ is independent of $L$ and $S$ in this case (since the fish have no psychic powers), it's clear that $p(I \mid L)=p(I \mid S)$. And we've already agreed in this case that $p(E \mid L \wedge I)=p(E \mid S \wedge I)$, so LP** entails that we have no evidence for $L$ over $S$, which is the right result. And in general, whenever $I$ is probabilistically independent of the relevant hypotheses, $p(E \mid H 1 \wedge I)>$ $p(E \mid H 2 \wedge I)$ iff $p(E \wedge I \mid H 1)>p(E \wedge I \mid H 2)$, so LP** will never issue in a different result than LP* as long as $p(I \mid H 1)=p(I \mid H 2)$. Thus, LP** respects the observation made above that in cases where $I$ is probabilistically independent of $H 1$ and $H 2, \mathrm{LP}^{*}$ is true; thus, LP** explains the apparent plausibility of LP*.

In the Firing Squad case, LP** entails that we should compare $p(E \mid D \wedge I) \times$ $p(I \mid D)$ and $p(E \mid C \wedge I) \times p(I \mid C)$. As already observed, $p(E \mid D \wedge I)=p(E \mid C \wedge I)$, so $p(E \mid D \wedge I) \times p(I \mid D)>p(E \mid C \wedge I) \times p(I \mid C)$ iff $p(I \mid D)>p(I \mid C)$. And since $p(I \mid D)>p(I \mid C)$ in the Firing Squad case, we have evidence for $D$ over $C$, which is again the right result.

LP** also solves - or, perhaps dissolves - the Line-Drawing Problem. Recall that the Line-Drawing Problem was that if we include too much information about the process of observation in $I$, we'll end up with the absurd result that $E$ can't be any evidence for anything. That problem resulted from a picture where we're comparing $p(E \mid H 1 \wedge I)$ and $p(E \mid H 2 \wedge I)$ (as LP* requires), and worrying about how to characterize $I$ in a way that didn't include so much information that these quantities would always be equal (as they would be if $I$ entails $E$, for then $p(E \mid H 1 \wedge I)=p(E \mid H 2 \wedge I)=1)$. But once we switch to comparing $p(E \wedge I \mid H 1)$ and $p(E \wedge I \mid H 2)$ as LP** requires, then there's no longer any concern about "including too much information" in $I$. For suppose that $I$ entails $E$ by being equivalent to the conjunction of $E$ with some additional information $J$. Then $p(E \wedge I \mid H 1)=p(E \wedge E \wedge J \mid H 1)=p(E \wedge J \mid H 1)$ and $p(E \wedge I \mid H 2)=p(E \wedge E \wedge J \mid H 2)=p(E \wedge J \mid H 2)$. So LP** results in the same likelihoods (and thus the same likelihood ratio) regardless of whether we conjoin the $E$-entailing $I$ to the left-hand side or whether we just conjoin the information in $I$ that "goes beyond" $E$-i.e., $J$ - to the left-hand side. As a result, there's no worry about changing the values of the likelihoods by "including too much about the process of observation" in $I$; even if $I$ entails $E$, conjoining $I$ to the left-hand side of the likelihoods will have the same effect as conjoining $J$ to the left-hand side. So even if we "build $E$ into $I$," no threat of triviality looms. 


\section{What My Proposal Says About the FTA}

According to the FTA, $E$ is evidence for $D$ over $C$ because $p(E \mid D)>p(E \mid C)$. According to the Objection from Anthropic Bias, $E$ fails to be evidence for $D$ over $C$ because (where $I=$ "I'm around at $t$ to make observations") $p(E \mid D \wedge I)=$ $p(E \mid C \wedge I)$.

According to LP**, the relevant question to ask is whether $p(E \wedge I \mid D)>$ $p(E \wedge I \mid C)$. And it's obvious that $p(E \wedge I \mid D)>p(E \wedge I \mid C)$, since I'm much likelier to be alive and observing a life-sustaining universe at $t$ if $D$ is true than if $C$ is true.

I conclude that Objection from Anthropic Bias is not a successful response to the Fine-Tuning Argument.

\section{References}

[Colyvan et al 2005] Colyvan, M., J. Garfield, and G. Priest. (2005). "Problems with the Argument from Fine Tuning," Synthese 145, pp. 325-338.

[Eddington 1939] Eddington, A. (1939). The Philosophy of Physical Science, Cambridge University Press.

[Juhl 2005] Juhl, C. (2005). "Fine-Tuning, Many Worlds, and the 'Inverse Gambler's Fallacy,'” Nous 39, pp. 337-347.

[Manson and Thrust 2003] Manson, N. and M. Thrush. (2003). "Fine-Tuning, Multiple Universes, and the 'This Universe' Objection," Pacific Philosophical Quarterly 84, pp. 67-83.

[McGrew et al 2001] McGrew, L., T. McGrew, and E. Vestrup. (2001). "Probabilities and the Fine-Tuning Argument: a Sceptical View," Mind 110, pp. $1027-1038$.

[Sober 2002] Sober, E. (2002). "Intelligent Design and Probability Reasoning," International Journal for the Philosophy of Religion 52, pp. 65-80.

[Sober 2004] Sober, E. (2004). "The Design Argument," in W. E. Mann (ed.), Blackwell Guide to the Philosophy of Religion, Blackwell Publishers, pp. 117147.

[Sober 2009] Sober, E. (2009). "Absence of Evidence and Evidence of Absence: Evidential Transitivity in Connection with Fossils, Fishing, Fine-tuning, and Firing squads," Philosophical Studies 143, pp. 63-90

Weisberg 2005Weisberg, J. (2005). "Firing Squads and Fine-Tuning: Sober on the Design Argument," British Journal for the Philosophy of Science 56, pp. 809-821.

[White 2000] White, R. (2000). "Fine-Tuning and Multiple Universes," Nous 34, pp. 260-276. 